\title{
Interferon Therapy and Prevention of Hepatocellular Carcinoma in Hepatitis $\mathbf{C}$
}

\author{
Amit G. Singal $\cdot$ Jorge A. Marrero
}

Received: 17 January 2012/ Accepted: 18 January 2012/Published online: 22 February 2012

(C) Springer Science+Business Media, LLC 2012

Hepatocellular carcinoma (HCC) is the third leading cause of cancer-related death worldwide and one of the leading causes of death among patients with cirrhosis. Its incidence is expected to continue increasing over the next 20 years and to peak around 2030 [1]. Although hepatitis B remains the most common risk factor worldwide, chronic hepatitis $\mathrm{C}$ virus (HCV) infection is the driving force for the increased incidence of $\mathrm{HCC}$ in Western countries and Japan [1]. Therefore, strategies aimed at eliminating the virus may provide opportunities for primary and secondary prevention of the development of HCC.

The first evidence of primary prevention of HCC was from a small randomized controlled trial with $90 \mathrm{HCV}$ infected patients in which interferon (IFN) treatment was associated with a significant reduction in the incidence of HCC [2]. The beneficial effects of IFN therapy were thought to be related to its anti-proliferative, anti-angiogenic, and/or anti-tumor effects [3, 4]. Since that time, several studies have replicated the findings of a reduction in HCC risk among patients treated with IFN, particularly patients who achieved a sustained viral response (SVR). A meta-analysis found that SVR was associated with a $79 \%$ (95\% CI 0.73-0.84) reduction in the risk of development of HCC by patients with HCV-related cirrhosis [5]. The primary preventive effect of IFN therapy may be even greater among non-cirrhotic $\mathrm{HCV}$ patients, given that SVR at

\footnotetext{
A. G. Singal

Division of Digestive and Liver Diseases, Department of Internal Medicine, UT Southwestern Medical Center, Dallas, TX, USA

\section{J. A. Marrero $(\square)$}

Division of Gastroenterology, Department of Internal Medicine, University of Michigan, 3912 Taubman Center,

SPC 5362, Ann Arbor, MI 48109, USA

e-mail: jmarrero@umich.edu
}

earlier stages of fibrosis can prevent the development of cirrhosis.

Given that the effect of IFN therapy was primarily seen among patients who had SVR, the utility of maintenance pegylated interferon (PEG-IFN) to prevent disease progression and HCC among non-responders was evaluated. Several randomized trials (HALT-C, COPILOT, and EPIC) have since demonstrated that maintenance PEG-IFN fails to prevent $\mathrm{HCC}$ in patients with $\mathrm{HCV}$-related cirrhosis among non-responders [6-8].

Patients with HCC who are diagnosed at an early stage can be effectively treated by liver transplantation, surgical resection, or local ablative therapy [9]. Although liver transplantation effectively treats both the HCC and replaces the underlying cirrhotic liver, it is not possible for all patients, given rigorous selection criteria and a shortage of organs. Surgical resection and local ablative therapy are able to achieve high cure rates, but these patients remain at high risk of developing recurrent or de-novo cancers. Recurrence 5 years after resection of primary tumors is reported to be approximately 50\% [10]. Early recurrence seems to be primarily driven by metastases and is associated with factors such as microvascular invasion and AFP level. Late recurrence, i.e., that occurring after 2 years, often appears as de-novo lesions and is associated with factors such as hepatitis activity and persistent HCV viremia [11]. Given the widespread implementation of HCC surveillance programs and increasing burden of HCC, a greater proportion of patients is expected to be diagnosed during early stages and be suitable for either resection or local ablation. Given the associated high incidence of recurrent or de-novo tumors, further studies that address prevention of HCC recurrence are crucial.

Several studies, including five randomized controlled trials and subsequent meta-analysis, have demonstrated 
that $\mathrm{HCV}$ treatment after resection or ablation (i.e., secondary prevention) can significantly reduce $\mathrm{HCC}$ recurrence in patients with $\mathrm{HCV}$ cirrhosis [12]. Although most studies used standard IFN monotherapy, one study included patients who received IFN and ribavirin (RBV) combination therapy and one assessed PEG-IFN monotherapy. $\mathrm{HCV}$ treatment seems to be fairly well tolerated by these patients, with $35-100 \%$ of patients receiving over $80 \%$ of the IFN doses, and is associated with SVR ranging from 4 to $69 \%$ across studies. For patients with SVR, incidence of $\mathrm{HCC}$ recurrence is approximately $35 \%$, significantly lower than the $61 \%$ recurrence among non-responders ( $p=0.005$ ) [12]. Furthermore, receipt of IFN therapy was associated with significantly improved 5-year survival (OR $0.40,95 \%$ CI $0.23-0.70)$.

The study by Miyatake et al. [21]. in this journal explores an interesting but understudied aspect of the role of HCV treatment in the secondary prevention of HCC. Although several studies have highlighted the benefit of $\mathrm{HCV}$ treatment for primary prevention of HCC and its role in the secondary prevention of recurrence in patients who have developed HCC, only a few studies had previously studied the effect of $\mathrm{HCV}$ treatment on recurrence before the development of HCC. In this study, 124 patients who received standard IFN monotherapy before the development of HCC (17 patients with SVR and 107 without SVR) were compared with 271 patients who had not previously received IFN therapy. Of these 395 patients, 103 were treated with surgical resection and 292 with local ablative therapy. The choice of curative therapy did not significantly differ between IFN-treated patients and IFNuntreated patients $(p=0.10)$, although surgical resection was performed significantly more often for those who achieved SVR than for non-SVR patients $(p=0.002)$. Although incidence of first HCC recurrence was similar among the groups $(36,47$, and $48 \%$ at 2 years for SVR, non-SVR, and untreated patients, respectively, $p=0.41$ ), incidence of second $\mathrm{HCC}$ recurrence was significantly lower among patients with SVR than among the non-SVR group and among the patients who had not previously received IFN therapy. Incidence of second $\mathrm{HCC}$ recurrence at 2 years was $10 \%$ among those with SVR compared with $69 \%$ among those without SVR $(p=0.003)$ and $70 \%$ in the IFN-untreated group $(p=0.006)$. The authors found that overall survival was also higher for patients with SVR than for non-SVR and untreated patients, with response to previous therapy being the only independent factor associated with survival $(p<0.001)$.

It is interesting to note in this study that initial recurrence was not significantly different between the groups, despite patients' undergoing IFN therapy a median of 7.2 (range 0.8-17.4) years before the development of HCC. The authors postulate that carcinogenic potential in SVR patients may be gradually attenuated because of eradication of the HCV infection, whereas it may increase in those with non-SVR, because of the persistence of $\mathrm{HCV}$ viremia. This is unlikely to explain this lack of difference, given that most patients were treated several years before the initial curative therapy. This lack of difference emphasizes that early recurrence is driven by intra-hepatic metastases and is more strongly associated with tumor factors than viral factors. Similar to the trials after HCC therapy, the benefit of HCV therapy and SVR is primarily limited to late recurrence, which is more likely to be de-novo tumors [1113].

This study supports the efficacy of IFN therapy before the development of HCC and its effects on tumor recurrence. Previous studies have analyzed how receipt of IFN therapy [14, 15] and biochemical response [16, 17] before HCC development affect post-treatment recurrence, but this is the first to assess the effect of IFN therapy according to viral response. However, there are important shortcomings of this study that must be taken into account. First, this was a retrospective cohort study which leads to specific biases. Second, patients with SVR had significantly lower Child-Pugh scores than non-SVR untreated patients and therefore, may have been at lower baseline risk of late HCC recurrence. Furthermore, information on other factors known to affect late recurrence, including fibrosis severity and grade of liver disease, were not available for all patients and not included in the multivariate analysis. Finally, these patients were treated between 1995 and 2006 and received standard IFN monotherapy instead of currently available therapy, so SVR was only achieved for a minority of patients. Future prospective studies, preferably randomized controlled trials, are necessary to account for these confounders and to assess HCC recurrence with addition of RBV and protease inhibitors to IFN therapy.

Another important aspect of this study is that only $38 \%$ of patients who underwent resection and $29 \%$ of patients who underwent ablation actually received IFN therapy before HCC development. This study and others indicate that only a minority of patients with chronic HCV infection are being offered antiviral therapy, with many patients being unaware of the diagnosis [18]. If this trend continues, $<15 \%$ of liver-related morbidity and mortality caused by $\mathrm{HCV}$ would be prevented. Introduction of telaprevir and boceprevir now offers the possibility of greater achievement of SVR with shorter duration of treatment for treatment-naïve and for non-responders to previous antiviral treatment [19, 20].

With growing evidence in support of the efficacy of HCV therapy in HCC prevention, including this study by Miyatake, it is becoming increasingly clear that providers must make a strong effort to offer $\mathrm{HCV}$ treatment to appropriate candidates, particularly those with cirrhosis or 
advanced fibrosis who are at high risk of developing HCC. Increased treatment and introduction of more potent antiviral agents may not only help prevent future HCC cases but also help improve outcomes for patients who develop cirrhosis and HCC.

\section{References}

1. El-Serag HB, Rudolph KL. Hepatocellular carcinoma: epidemiology and molecular carcinogenesis. Gastroenterology. 2007; 132:2557-2576.

2. Nishiguchi S, Kuroki T, Nakatani S, et al. Randomised trial of effects of interferon-alpha on incidence of hepatocellular carcinoma in chronic active hepatitis $\mathrm{C}$ with cirrhosis. Lancet. 1995;346:1051-1055.

3. Singh RK, Gutman M, Bucana CD, et al. Interferons alpha and beta down-regulate the expression of basic fibroblast growth factor in human carcinomas. Proc Natl Acad Sci USA. 1995; 92:4562-4566.

4. Lai CL, Lau JY, Wu PC, et al. Recombinant interferon-alpha in inoperable hepatocellular carcinoma: a randomized controlled trial. Hepatology. 1993;17:389-394.

5. Singal AG, Volk ML, Jensen D, et al. A sustained viral response is associated with reduced liver-related morbidity and mortality in patients with hepatitis C virus. Clin Gastroenterol Hepatol. 2010;8:280-288, 288 e281.

6. Shiffman ML, Di Bisceglie AM, Lindsay KL, et al. Peginterferon alfa-2a and ribavirin in patients with chronic hepatitis $\mathrm{C}$ who have failed prior treatment. Gastroenterology. 2004;126:10151023; Discussion 1947.

7. Bruix J, Poynard T, Colombo M, et al. Maintenance therapy with peginterferon alfa-2b does not prevent hepatocellular carcinoma in cirrhotic patients with chronic hepatitis C. Gastroenterology. 2011;140:1990-1999.

8. Afdhal NH, Levine R, Brown RJ, et al. Colchicine versus peginterferon alfa $2 \mathrm{~b}$ long-term therapy: results of the 4 year COPILOT trial. In: 43rd Annual Meeting of the European Association for the Study of the Liver. Milan, Italy; 2008.

9. Bruix J, Sherman M. Management of hepatocellular carcinoma: an update. Hepatology. 2011;53:1020-1022.
10. Margarit C, Escartin A, Castells L, et al. Resection for hepatocellular carcinoma is a good option in Child-Turcotte-Pugh class A patients with cirrhosis who are eligible for liver transplantation. Liver Transpl. 2005;11:1242-1251.

11. Imamura H, Matsuyama Y, Tanaka E, et al. Risk factors contributing to early and late phase intrahepatic recurrence of hepatocellular carcinoma after hepatectomy. J Hepatol. 2003; 38:200-207.

12. Singal AK, Freeman DH Jr, Anand BS. Meta-analysis: interferon improves outcomes following ablation or resection of hepatocellular carcinoma. Aliment Pharmacol Ther. 2010;32:851-858.

13. Lok AS, Everhart JE, Wright EC, et al. Maintenance peginterferon therapy and other factors associated with hepatocellular carcinoma in patients with advanced hepatitis C. Gastroenterology. 2011;140:840-849; Quiz e812.

14. Komorizono Y, Sako K, Yamasaki N, et al. Outcome of patients with hepatitis $\mathrm{C}$ virus-related hepatocellular carcinoma occurring after interferon therapy. Anticancer Res. 2002;22:3573-3578.

15. Kubo S, Nishiguchi S, Hirohashi K, et al. Influence of previous interferon therapy on recurrence after resection of hepatitis $\mathrm{C}$ virus-related hepatocellular carcinoma. Jpn J Cancer Res. 2001; 92:59-66.

16. Uenishi T, Kubo S, Hirohashi K, et al. Relationship between response to previous interferon therapy and postoperative recurrence of hepatitis C virus-related hepatocellular carcinoma. Hepatol Res. 2002;24:404-412.

17. Uenishi $\mathrm{T}$, Nishiguchi S, Tamori A, et al. Influence of interferon therapy on outcome after surgery for hepatitis $\mathrm{C}$ virus-related hepatocellular carcinoma. Hepatol Res. 2006;36:195-200.

18. Volk ML, Tocco R, Saini S, Lok AS. Public health impact of antiviral therapy for hepatitis $\mathrm{C}$ in the United States. Hepatology. 2009;50:1750-1755.

19. McHutchison JG, Everson GT, Gordon SC, et al. Telaprevir with peginterferon and ribavirin for chronic HCV genotype 1 infection. N Engl J Med. 2009;360:1827-1838.

20. Poordad F, McCone J Jr, Bacon BR, et al. Boceprevir for untreated chronic HCV genotype 1 infection. $N$ Engl J Med. 2011;364:1195-1206.

21. Miyatake H, Kobayashi Y, Iwasaki Y, et al. Effect of previous interferon treatment on outcome after curative treatment for hepatitis C virus-related hepatocellular carcinoma. Dig Dis Sci. doi:10.1007/s10620-011-1934-1. 